\title{
Gestione iniziale della terapia con insulina basale nel paziente diabetico
}

\author{
Francesco Giorgino $^{1} \cdot$ Sergio Di Molfetta ${ }^{1}$. Irene Caruso ${ }^{1}$
}

Accettato: 21 giugno 2020 / Pubblicato online: 16 novembre 2020

(c) The Author(s) 2020

\section{Introduzione}

Un buon controllo glicemico è essenziale per ridurre l'incidenza e la progressione delle complicanze micro- e macrovascolari nelle persone con diabete mellito di tipo 1 (DM1) e di tipo 2 (DM2). Tuttavia, gran parte dei pazienti mantiene un controllo insoddisfacente per lunghi periodi di tempo [1]. L'introduzione di un'insulina basale è una valida opzione per l'intensificazione del trattamento nelle persone con DM2. Tuttavia, definire una dose di partenza appropriata e fornire schemi per l'auto-titolazione dell'insulina basale è importante anche nelle persone con DM1 per raggiungere in sicurezza gli obiettivi di cura della malattia.

\section{Diabete mellito di tipo 2}

Le linee guida internazionali raccomandano l'impiego dell'insulina basale in pazienti affetti da DM2 che non raggiungono gli obiettivi di trattamento nonostante duplice/triplice terapia orale e/o con gli agonisti del recettore del GLP-1 o in presenza di grave scompenso glicometabolico con sintomi/segni di deficit insulinico (Fig. 1) [1]. In Italia, circa il $30 \%$ dei pazienti affetti da DM2 fa uso di insulina, da sola o in associazione ad altri ipoglicemizzanti (Annali AMD 2018).

Le formulazioni di insulina basale oggi disponibili sono l'insulina intermedia NPH, sempre meno usata, e gli ana-

$凶$ F. Giorgino

francesco.giorgino@uniba.it

1 Sezione di Medicina Interna, Endocrinologia, Andrologia e Malattie Metaboliche, Dipartimento dell'Emergenza e dei Trapianti di Organi, Università degli Studi di Bari Aldo Moro, Bari, Italia loghi ricombinanti dell'insulina umana di prima (detemir e glargine U-100) o seconda (degludec e glargine U-300) generazione. L'insulina intermedia NPH ha durata d'azione più breve rispetto alle altre formulazioni e il suo impiego è gravato da un maggior rischio di ipoglicemia. L'insulina detemir, invece, determina un minore incremento ponderale ma richiede a volte una doppia somministrazione giornaliera. Le insuline di seconda generazione sono caratterizzate da una durata d'azione più lunga, da un profilo farmacocinetico con picco meno evidente e da minore variabilità di azione da un giorno all'altro. Trial randomizzati controllati e studi di Real World Evidence (RWE) hanno dimostrato che, a parità di efficacia, l'impiego di insuline basali di seconda generazione consente di ridurre il rischio di ipoglicemia, che costituisce uno dei principali ostacoli all'intensificazione terapeutica [1].

La dose iniziale consigliata di insulina basale è di 10 UI/die (o 0,1-0,2 UI/kg/die), ma la prima prescrizione deve necessariamente essere seguita da una fase di titolazione, della durata di 8-12 settimane, durante la quale ci si prefigge di raggiungere il target personalizzato di glicemia a digiuno (FPG), solitamente compreso fra 80 e $130 \mathrm{mg} / \mathrm{dl}$, evitando ipoglicemie [1]. La titolazione può essere gestita dal diabetologo oppure dal paziente stesso, secondo algoritmi di autotitolazione. Le evidenze cliniche sostengono la maggiore efficacia delle strategie di auto-titolazione e, a differenza degli studi condotti con glargine U-100, lo studio TAKE CONTROL e lo studio italiano ITAS [2], condotti con glargine U-300, non hanno mostrato differenze nell'incidenza di ipoglicemie fra i due gruppi. Gli schemi di titolazione proposti sono molteplici e prevedono la riduzione o l'incremento di 2-3 UI (o loro multipli) di insulina basale ogni 3-7 giorni in base ai valori di FPG registrati dal paziente nei giorni precedenti $[1,3]$. 
Fig. 1 Guida

all'intensificazione terapeutica con insulina basale nel paziente affetto da diabete mellito di tipo 2 (DM2). GLP-1RA, agonisti del recettore del GLP-1

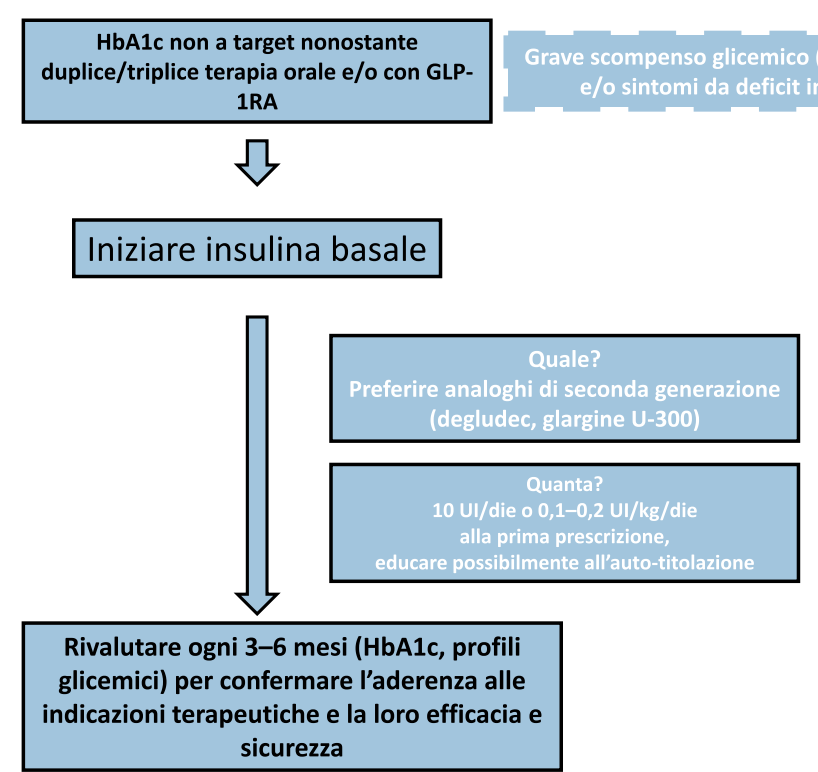

Fig. 2 Proposta di algoritmo di trattamento in pazienti adulti non in gravidanza affetti da diabete mellito di tipo 1 (DM1) [5]. $Q D$, una volta al giorno; $B I D$, due volte al giorno; $T I D$, tre volte al giorno

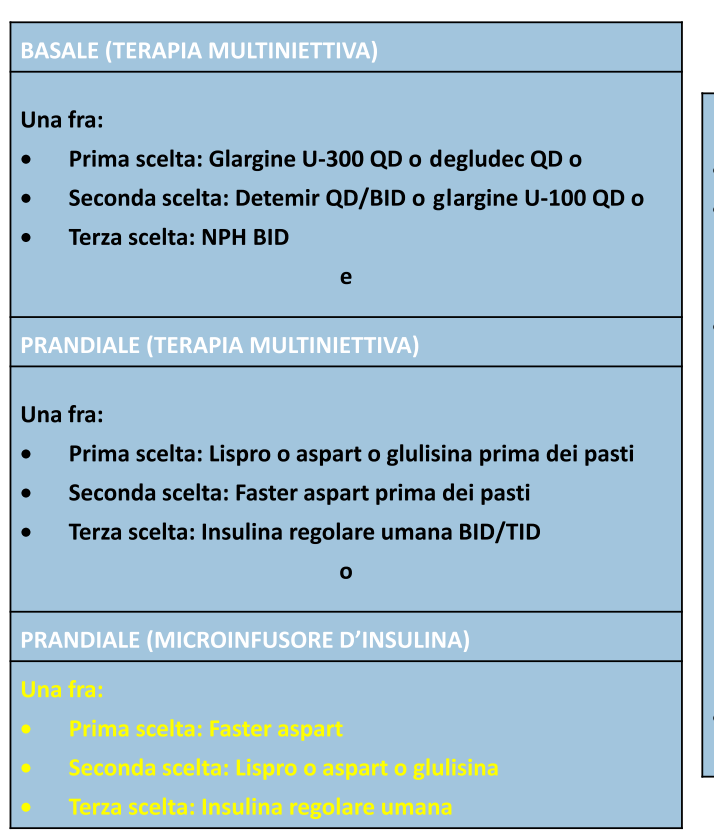

\section{Diabete mellito di tipo 1}

Nelle persone con DM1, schemi di terapia basal-bolus ( $\geq 3$ iniezioni di insulina al giorno o, meno frequentemente, infusione sottocutanea continua di insulina con un microinfusore) sono impiegati sin dall'epoca della diagnosi di malattia per mimare la secrezione fisiologica dell'insulina. Il fabbisogno insulinico giornaliero può essere stimato a partire dal peso corporeo ed è compreso fra 0,4 e $1,0 \mathrm{UI} / \mathrm{kg} / \mathrm{die}$ nella maggior parte degli individui. In particolare, le società scientifiche suggeriscono una dose insulinica di partenza pari a $0,5 \mathrm{UI} / \mathrm{kg} / \mathrm{die}$ nei pazienti con DM1 in buon controllo metabolico [4]. Nei pazienti di nuova diagnosi, la dose totale richiesta è spesso inferiore come conseguenza del recupero parziale della funzione $\beta$-cellulare che può avvenire dopo l'inizio della terapia insulinica; in questi casi è consigliata una dose di partenza più bassa $(0,3-0,4 \mathrm{UI} / \mathrm{kg} / \mathrm{die})$ per ridurre il rischio di ipoglicemia [5]. In genere, il 40-60\% della dose totale giornaliera è somministrata come insulina basale e la parte rimanente è suddivisa in 2-4 dosi prandiali in base al numero dei pasti e al loro contenuto di carboidrati.

Gli analoghi di seconda generazione dell'insulina basale (degludec e glargine U-300) sono da considerare di prima scelta nei pazienti in terapia multiniettiva, in quanto garantiscono una riduzione degli episodi ipoglicemici (soprattutto notturni) e una minore variabilità glicemica a parità di efficacia nel ridurre i livelli di HbA1c (Fig. 2) [5]. 
Anche nelle persone con DM1, l'aggiustamento delle dosi di insulina è guidato dai risultati dell'automonitoraggio domiciliare della glicemia. In particolare, la titolazione dell'insulina basale è fatta osservando la FPG dopo aver sistemato il bolo della cena (glicemia prima di andare a letto compresa fra 100 e $130 \mathrm{mg} / \mathrm{dl}$ ). Un aumento della glicemia $>30 \mathrm{mg} / \mathrm{dl}$ durante la notte deve indurre a un aumento della dose di insulina basale, mentre una riduzione della glicemia della stessa entità rende opportuna una riduzione di dose [6].

\section{Conclusione}

Nonostante l'introduzione di un'insulina basale, più del $70 \%$ dei pazienti non ottiene un buon controllo glicemico. La paura dell'ipoglicemia e dell'incremento ponderale, la scarsa motivazione e l'incapacità a titolare la dose in maniera appropriata sono i principali ostacoli al raggiungimento degli obiettivi di trattamento e la loro gestione è parte integrante del percorso di cura. Una maggiore attenzione all'educazione terapeutica e l'utilizzo di App per la titolazione attiva delle dosi potrebbero favorire l'aderenza al trattamento.

Funding Note Open access funding provided by Università degli Studi di Bari Aldo Moro within the CRUI-CARE Agreement.

Conflitto di interesse L'autore Francesco Giorgino dichiara di aver ricevuto compensi per collaborazione scientifica da AstraZeneca, Boehringer Ingelheim, Eli Lilly, Lifescan, Merck Sharp \& Dohme, Novo Nordisk, Roche Diabetes Care e Sanofi Aventis. L'autore Sergio Di Molfetta dichiara di aver ricevuto compensi per collaborazione scientifica da Roche Diabetes Care. L'autrice Irene Caruso dichiara di non avere conflitti d'interesse.

Consenso informato Lo studio presentato in questo articolo non ha richiesto sperimentazione umana.

Studi sugli animali Gli autori di questo articolo non hanno eseguito studi sugli animali.
Nota della casa editrice Springer Nature rimane neutrale in riguardo alle rivendicazioni giurisdizionali nelle mappe pubblicate e nelle affiliazioni istituzionali.

Open Access This article is licensed under a Creative Commons Attribution 4.0 International License, which permits use, sharing, adaptation, distribution and reproduction in any medium or format, as long as you give appropriate credit to the original author(s) and the source, provide a link to the Creative Commons licence, and indicate if changes were made. The images or other third party material in this article are included in the article's Creative Commons licence, unless indicated otherwise in a credit line to the material. If material is not included in the article's Creative Commons licence and your intended use is not permitted by statutory regulation or exceeds the permitted use, you will need to obtain permission directly from the copyright holder. To view a copy of this licence, visit http://creativecommons.org/licenses/by/4.0/.

\section{Bibliografia}

1. Khunti K, Giorgino F, Berard L et al (2019) The importance of the initial period of basal insulin titration in people with diabetes. Diabetes Obes Metab 22(5):722-733

2. Bonadonna RC, Giaccari A, Buzzetti R et al (2020) Comparable efficacy with similarly low risk of hypoglycaemia in patient- vs physician-managed basal insulin initiation and titration in insulinnaïve type 2 diabetic subjects: the Italian Titration Approach Study (ITAS). Diabetes Metab Res Rev 36(6):e3304

3. Berard L, Antonishyn N, Arcudi K et al (2018) Insulin matters: a practical approach to basal insulin management in type 2 diabetes. Diabetes Ther 9(2):501-519

4. Peters A, Laffel L (2013) American Diabetes Association/JDRF type 1 diabetes sourcebook. American Diabetes Association, Alexandria

5. Janež A, Guja C, Mitrakou A et al (2020) Insulin therapy in adults with type 1 diabetes mellitus: a narrative review. Diabetes Ther 11(2):387-409

6. Peters A, Wood J (2018) The type 1 diabetes self-care manual: a complete guide to type 1 diabetes across the lifespan for people with diabetes, parents, and caregivers. American Diabetes Association, Arlington. http://main.diabetes.org/dorg/PDFs/living-withdiabetes/T1DSelfCareManual.pdf. Accessed on 21 June 2020 